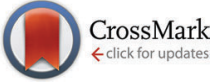

Cite this: Phys. Chem. Chem. Phys., 2016, 18, 13897

Received 3rd March 2016, Accepted 19th April 2016

DOI: $10.1039 / c 6 c p 01487 a$

www.rsc.org/pccp

\title{
Controlled rippling of graphene via irradiation and applied strain modify its mechanical properties: a nanoindentation simulation study $\dagger$
}

\author{
J. Martinez-Asencio, ${ }^{a}$ C. J. Ruestes, ${ }^{b}$ E. M. Bringa ${ }^{b}$ and M. J. Caturla*a
}

\begin{abstract}
Ripples present in free standing graphene have an important influence on the mechanical behavior of this two-dimensional material. In this study, we show through nanoindentation simulations, how out-ofplane displacements can be modified by strain, resulting in softening of the membrane under compression and stiffening under tension. Irradiation also induces changes in the mechanical properties of graphene. Interestingly, compressed samples, irradiated at low doses are stiffened by the irradiation, whereas the samples under tensile strain do not show significant changes in their mechanical properties. These simulations indicate that vacancies produced by the energetic ions cannot be the ones directly responsible for this behavior. However, changes in roughness induced by the momentum transferred from the energetic ions to the membrane, can explain these differences. These results provide an alternative explanation to recent experimental observations of the stiffening of graphene under low dose irradiation, as well as the paths to tailor the mechanical properties of this material via applied strain and irradiation.
\end{abstract}

\section{Introduction}

Graphene is a single layer of graphite, an atom thick allotrope of carbon arranged in a honeycomb lattice, which presents $\mathrm{sp}^{2}$ hybridization. This singular stable structure exhibits some unique physical properties, such as excellent electronic transport, ${ }^{1,2}$ thermal conductivity, ${ }^{3,4}$ optical $^{5,6}$ and mechanical response, ${ }^{7}$ especially for its elasticity and intrinsic strength features. All these make graphene one of the most promising and attractive materials of recent years. Therefore, it is being studied for a wide variety of applications. For instance, transistors, ${ }^{8-10}$ transparent electronic materials ${ }^{11}$ and sensors in nanoelectronics, ${ }^{12}$ cancer therapy ${ }^{13}$ in medicine, solar cells ${ }^{14}$ in energy storage industry and even conducting ink ${ }^{15,16}$ manufacturing, are some of many possible uses of this promising material.

One method of altering ${ }^{17}$ the abovementioned physical and chemical properties, and possibly enhancing them, consists of studying a graphene layer with impurities or defects such as adatoms, dislocations or vacancies. There are many ways to produce defects on a sample, ${ }^{18,19}$ but a simple and efficient way

\footnotetext{
${ }^{a}$ Dept. Física Aplicada, Facultad de Ciencias, Fase II, Universidad de Alicante, Alicante E-03690, Spain. E-mail: mj.caturla@ua.es

${ }^{b}$ CONICET and Facultad de Ciencias Exactas y Naturales, Universidad Nacional de Cuyo, Mendoza 5500, Argentina

$\dagger$ Electronic supplementary information (ESI) available. See DOI: 10.1039/ с6ср01487a
}

to produce them in a controlled manner is by ion bombardment. For these methods to be efficient and feasible, it is important to understand the type of defects produced under the different irradiation conditions such as the irradiation type (electrons or ions), temperature, energy and dose. The tuning energy and dose can lead to the formation of nanopores ${ }^{20,21}$ and some stable structures such as monoatomic chains. ${ }^{22}$ The damage produced by irradiation in supported graphene, as well as multilayer graphene has also been studied by several authors both experimentally and computationally (see for example ref. 23-26).

Numerous studies of irradiation damage have been performed using molecular dynamics for electron ${ }^{27}$ or ion implantation. ${ }^{28-32}$ The irradiation of graphene with $\mathrm{C}$ ions ranging from $0.1 \mathrm{eV}$ to $100 \mathrm{keV}$ at different positions on the hexagonal lattice has been studied by Bellido and Seminario ${ }^{29}$ and a similar study was carried out with $\mathrm{Si}$ ions. ${ }^{30}$ An experiment combined with MD simulations using $\mathrm{Ar}^{+}$ions on graphene/Ir(111) was performed for different beam energies. ${ }^{33}$ In addition, an experimental study based on $\mathrm{Ar}^{+}$bombarded graphene on a $\mathrm{SiO}_{2}$ substrate was performed by Dobrik et al., ${ }^{34}$ wherein they concluded that the Fermi velocity is reduced in the presence of defects. Increasing the quantity of defects in graphene leads to weakening of its robustness, making it less stiff and degrading its mechanical properties. $^{35,36}$ More recent experiments, ${ }^{37}$ however, have shown that irradiation of suspended graphene using low energy Ar ions obtain increased stiffness of the graphene membrane when defect concentrations are around $0.1-0.2 \%$, reporting values of 
two-dimensional Young modulus $\left(E_{2 \mathrm{D}}\right)$ higher than for pristine graphene. ${ }^{7}$ Several explanations have been proposed for the mechanism of stiffening of graphene at such low defect concentrations. $^{37,38}$

One important phenomenon in two-dimensional systems such as graphene, which might have been overlooked, is the influence of the out-of-plane displacements present in these structures. It is well-known that graphene is not perfectly flat, but it forms ripples ${ }^{39,40}$ due to thermal fluctuations and its twodimensional nature. The structure of these ripples and their influence on the different properties of graphene, including elastic properties, ${ }^{41}$ has been the interest of many studies. ${ }^{42-44}$ Recent molecular dynamics simulations have shown that intrinsic ripples of graphene affect its elasticity, resulting in softening of this material. ${ }^{41}$

This study presents molecular dynamics simulations of the irradiation of suspended graphene membranes with $140 \mathrm{eV} \mathrm{Ar}$ ions for different values of the initial strain at $300 \mathrm{~K}$. We focus on the nature of those defects produced by the irradiation as a function of the dose and applied strain, as well as the changes in the ripple distribution and roughness. Nanoindentation simulations of the graphene membrane show how the mechanical properties of this material change for different conditions of the applied strain and irradiation.

\section{Methods}

Classical molecular dynamics (MD) simulations of irradiation have been performed using the LAMMPS code. ${ }^{45}$ A graphene drumhead of radius $75 \mathrm{~nm}$, which contained 674644 carbon atoms, was used for these calculations. The Tersoff/ZBL potential was used to simulate the $\mathrm{C}-\mathrm{C}$ interaction. This potential accounts for 3-body interactions using the Tersoff potential ${ }^{46}$ and also short-range interactions between atoms mimicking a Coulombic repulsive potential using a screening function described by the so-called ZBL potential, Ziegler-Biersack-Littmarck, ${ }^{47}$ which is needed for the short distance interactions that occur when performing irradiation simulations. The ZBL potential was then used for the Ar-C and Ar-Ar interactions. The Tersoff potential has been used by other authors to examine the damage produced in graphene. ${ }^{21,28,29,38,48}$

Fixed boundary conditions were considered in the outer layer of the drumhead. The regions were set as follows: an outermost annulus of $1 \mathrm{~nm}$ with fixed atoms, an adjacent annulus of $2 \mathrm{~nm}$ with a Langevin thermal bath and a dynamic region for the rest of the drumhead. In this way, we achieve simulating a suspended graphene flake with a cooling down region, so that a smooth transition between the dynamic region and the one with fixed atoms occur. The simulation volume is relaxed using the Polak-Ribière version of the conjugate gradient algorithm $^{49}$ so that any residual stress is removed. Simulations were also performed for different initial strains, from compressive to tensile, with values between $-0.25 \%$ and $0.25 \%$, respectively. For those simulations, wherein an initial strain is applied, the strain is produced by changing the simulation box size and remapping the atomic positions according to the new box size. The system is then relaxed for 3 ps in NPH ensemble at $P=0$ bars in the $x$ and $y$ axis and a temperature of $300 \mathrm{~K}$. The time step used in these simulations is $1 \mathrm{fs}$.

The simulation of irradiation of the graphene drumhead is set as follows. After the system is relaxed to the selected strain conditions, the sample was irradiated with one low energy $\mathrm{Ar}$ ion $(140 \mathrm{eV})$ every 5000 time steps and a total of 1000 ions were shot. In this case, a variable time step was considered to account for the short range interaction of the energetic atoms. The sample was irradiated perpendicularly within a circular region with a radius of $65 \mathrm{~nm}$ from its center with the irradiation point selected randomly within this region. The system was equilibrated for 35000 steps after the 1000 ion irradiation. The NVE ensemble during the irradiation was employed for the inner region of the drumhead. The simulations were performed at $300 \mathrm{~K}$.

One of the parameters analyzed is the roughness of the membranes and how it evolves during irradiation. The roughness was calculated as the average of the square of the distance in the $z$ direction (perpendicular to the membrane) with respect to the initial position, $z_{0}$, of all atoms in the membrane, $<\left(z-z_{0}\right)^{2}>$.

Defects were identified using OVITO $^{50}$ and classified as monovacancies, divacancies, and higher order vacancy clusters (more than two vacancies). A first nearest neighbor distance was considered to identify if vacancies belong to the same cluster.

A set of drumheads was selected for indentation simulations to assess the effect of strain and irradiation on their mechanical properties, namely, its elastic modulus. Indentation was performed using a spherical indenter tip modeled by a repulsive potential, as described by Kelchner et al. ${ }^{51}$ and the indenter repels all atoms that touch it so that the exerted force has a magnitude of

$$
F_{\mathrm{r}}=-K(r-R)^{2}
$$

where $K$ is a force constant, herein set to $10 \mathrm{eV} \AA^{-3}, r$ is the distance from the atom to the center of the indenter and $R$ is the radius of the indenter. The force was set to 0 for $r>R$. The indenter radius was set to $10 \mathrm{~nm}$.

Indentation was performed in displacement-controlled mode, similar to other MD studies, ${ }^{52}$ using a penetration rate of $5 \mathrm{~m} \mathrm{~s}^{-1}$. Penetration was restricted to a depth in the range of 5-7.5 nm, for a total simulation time of $1500 \mathrm{ps}$ and a $1 \mathrm{fs}$ timestep. Upon contact with the graphene flake, the reaction force between the flake and the indenter was computed every 0.1 ps to extract the load-penetration curves. Indentation was performed at $300 \mathrm{~K}$.

The literature review shows that for the derivation of the elastic modulus based on the nanoindentation loaddisplacement curves, the graphene membrane was modeled following the non-linear Föppl membrane theory. ${ }^{53}$ Lee et al. ${ }^{7}$ produced a fitting function for the treatment of nanoindentation load-displacement curves in agreement with the non-linear Föppl membrane theory. Namely,

$$
F(\delta)=\pi \sigma_{0}^{2 \mathrm{D}} \delta+q^{3} / a^{3} E_{2 \mathrm{D}} \delta^{3}
$$


where $\delta$ is the deflection, $\sigma_{0}^{2 \mathrm{D}}$ is the membrane pretension, $E_{2 \mathrm{D}}$ is the two-dimensional Young's modulus, $a$ is the radius of the membrane and $q$ is a correction factor for a Poisson's ratio $(\nu)$ other than one third and it takes the form of $q \sim 1.0491-$ $0.1462 \nu-0.15827 \nu^{2}$. Refer Komaragiri et al. ${ }^{54}$ for details on the determination of the $q$ function. It can be observed that the linear term of the equation captures the effects of the pretension, whereas the cubic term accounts for the effects of the bending stiffness. Using a least-square fitting of eqn (2) to the load-displacement curves, $\sigma_{0}^{2 \mathrm{D}}$ and $E_{2 \mathrm{D}}$ can be determined. A discussion of the $E_{2 \mathrm{D}}$ values obtained compared to experimental values is included in the ESI (S1) $\dagger$

\section{Results and discussion}

Fig. 1(a) shows the roughness of the membrane as a function of the applied strain before irradiation. Starting from the roughness value of the sample with no strain $(0 \%)$, we can observe that when a tensile strain is applied, the roughness decreases, reaching almost a steady value after a strain of about $0.1 \%$. When a compressive strain is applied, the roughness keeps increasing with increasing compression and the dependence is highly non-linear.

Nanoindentation simulations have been performed to obtain the $E_{2 \mathrm{D}}$ and sigma constants for different strains, following the procedure explained above. These two parameters were obtained from a fit to the curves of load as a function of displacement. For an example of these curves, observe Fig. S1 in the ESI. $\dagger$ Fig. 1(b) shows the values of $E_{2 \mathrm{D}}$ before irradiation as a function of the applied strain. Clearly, the strain plays an important role in the mechanical properties of graphene membranes. Compressive strain results in softening of the graphene membrane decreasing the value of the 2D Young's modulus, while graphene becomes stiffer under tension. This is in agreement with the results of Lee $e t a{ }^{41}$ showing that the presence of ripples results in the softening of graphene because two mechanisms occur: first, the ripples are smoothed and then the $\mathrm{C}-\mathrm{C}$ bond is stretched.

Roughness, as defined above, provides an average value of the fluctuations on the surface, but it is also interesting to observe the distribution of these fluctuations. Fig. 2 provides an image of the graphene membranes, wherein the colors represent the value of the out-of-plane coordinate of each atom ( $Z$ coordinate).
Three representative examples are given: Fig. 2(a) corresponds to a compressive strain of $-0.25 \%$, Fig. 2 (b) to a non-strained sample, and Fig. 2(c) to a tensile strain of $0.25 \%$. Note that the scale changes for each one of the figures.

This representation allows us to observe the wavelength of these ripples and the effect of strain on their distribution. The wavelength of the ripples decreases under compressive strain, showing a smaller distance between the maxima and minima of the ripples in the membrane (see Fig. 2(a) compared to Fig. 2(b), compressed and un-strained, respectively). The values of the maximum and minimum increase with respect to those from the sample without strain, with absolute values of approximately $3.5 \AA$ for a compressive strain of $-0.25 \%$ and approximately $2 \AA$ for the unstrained sample. When a tensile strain is applied, the wavelength increases significantly, with maxima and minima farther away from each other, as can be observed in Fig. 2(c). In addition, the values of these maxima and minima decrease, with absolute values of about $1.9 \AA$ for a $0.25 \%$ tensile strain. These changes, however, are much smaller than for a compressive strain, as already shown in Fig. 1. The observed ripples in our calculations are similar to the reported ripples in free-standing graphene measured experimentally. ${ }^{55}$

After relaxation, the sample is irradiated with $140 \mathrm{eV} \mathrm{Ar}^{+}$ ions following the procedure described in the Methods section. Irradiation produces vacancies that are mostly isolated, as well as divacancies and some higher order clusters at much lower concentrations. The efficiency of defect production is approximately $80 \%$, in which 80 vacancies are formed for every 100 ions. This is in good agreement with experimental measurements of López-Polín et al. ${ }^{37}$ For the doses studied herein, (highest dose of $7.5 \times 10^{12}$ ions per $\mathrm{cm}^{2}$ ) the number of vacancies increases linearly with dose, which indicates that the dose is low enough not to achieve an overlap of cascades. The total number of monovacancies is similar for all strains and doses. However, the number of divacancies is slightly higher when samples are under compressive strain and the efficiency of production of divacancies increases with the dose (for more information see ESI, $\dagger$ Fig. S2).

The changes in roughness with irradiation dose are presented in Fig. 3(a) for two applied strains, a compression of $-0.2 \%$ and a tension of $0.2 \%$. The differences in roughness
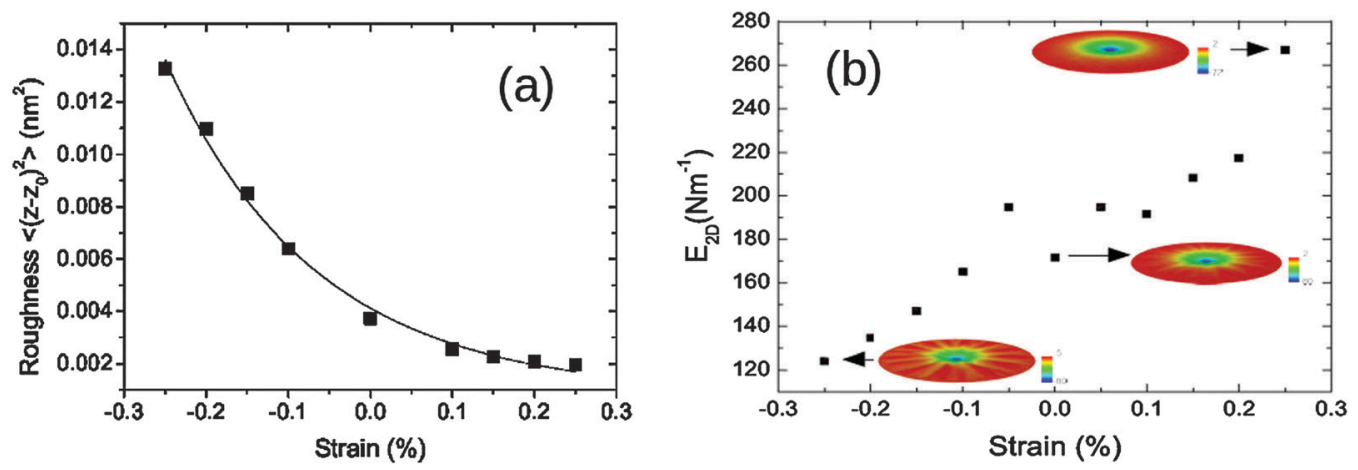

Fig. 1 (a) Roughness of the graphene membrane as a function of applied strain before irradiation and (b) $E_{2 D}$ obtained from nanoindentation simulations as a function of the applied strain. The insets show images of the simulations with colors representing the out-of-plane displacement (in $\AA$ ). 


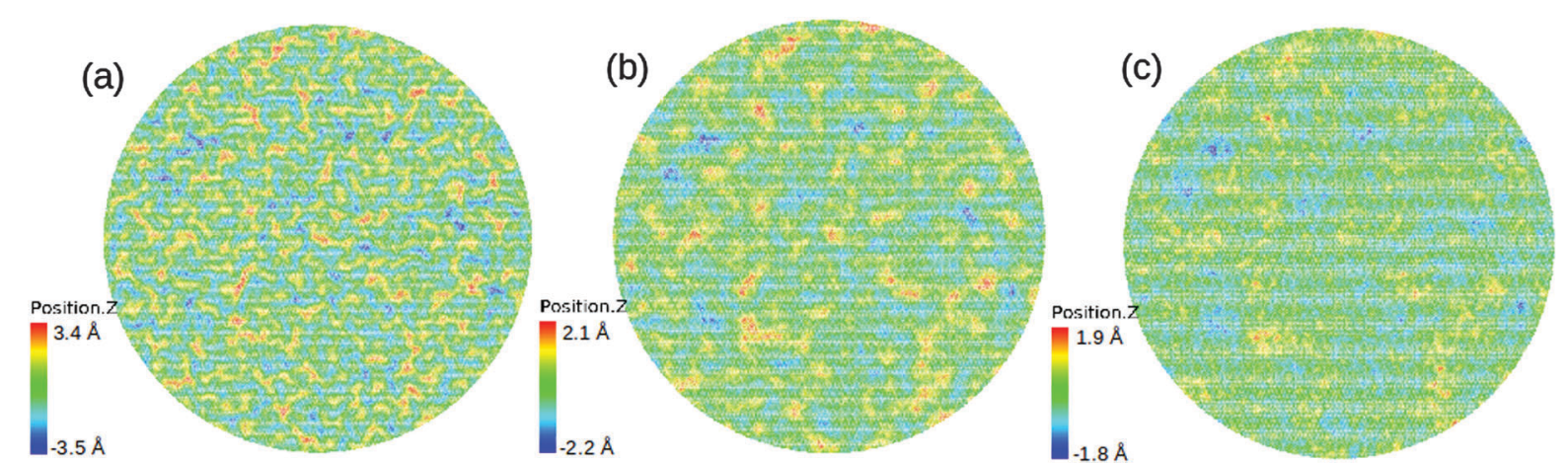

Fig. 2 Values of the $Z$ coordinate (in Angstroms) for a graphene membrane and different applied strains before irradiation (a) compressive $-0.25 \%$, (b) no strain and (c) tensile $+0.25 \%$.

induced by the irradiation are quite remarkable. While there are almost no changes when the sample is under tensile strain, roughness increases significantly with dose under a compressive strain. Note that changes in roughness induced by irradiation are over two orders of magnitude larger than those before irradiation for any given strain (see Fig. 1(a)). Fig. 3(b) includes the results of the $E_{2 \mathrm{D}}$ for the case of two compressive strains $(-0.25 \%,-0.1 \%)$, the sample without any initial strain, and two tensile strains $(0.1 \%$ and $0.25 \%)$. Interestingly, the dependence of the Young's modulus with the dose follows that of the roughness for the different strains: under tensile strain there is almost no change of the Young's modulus with dose, while under compression, the Young's modulus increases with dose, at very low doses, the $E_{2 \mathrm{D}}$ does not change significantly but it then becomes stiffer quite rapidly with the dose. When no strain is applied, there are almost no changes of the $E_{2 \mathrm{D}}$ with dose.

Note that the dependence of the $E_{2 \mathrm{D}}$ shown in Fig. 3(b) cannot be explained by the production of defects during irradiation alone because the number of defects increases linearly with dose for all strains applied or for the sample without strain. If the defects are the ones responsible for changes in the elastic modulus, the same dependence should be obtained independently of the applied strain, which is not the case here. However, the changes in the elasticity of the graphene membrane follow the dependence shown in Fig. 3(a) for the variation of the roughness with dose. When a tensile strain is applied, there are no changes in the roughness of the membrane, which results in an almost constant value of the elastic properties, when a compressive strain is considered, the roughness increases with dose with a non-linear dependence just like the elastic Young's modulus.

Interestingly, under irradiation, the compressed samples result in stiffer membranes, while samples under tension do not present significant differences in the elastic properties. This result seems to contradict those presented in Fig. 1 for a nonirradiated sample: compressed samples are softer than tensile ones. To clarify how radiation influences out-of-plane deformations in the membrane, we present in Fig. 4 the $Z$-coordinates after irradiation for the highest dose simulated: $7.5 \times 10^{12}$ ions per $\mathrm{cm}^{2}$. Fig. 4(a) corresponds to a compressive strain of $-0.25 \%$, Fig. 4 (b) to a non-strained sample and Fig. 4(c) to a tensile strain of $0.25 \%$, i.e., the same conditions of Fig. 2 but after irradiation. It is clear form these figures that the changes induced by irradiation on the out-of-plane deformation are significantly different from those induced only by strain. For the unstrained and compressed samples, irradiation produces a deep well in the membrane, mostly in the center of the sample, removing the ripple distribution existing initially, while under tension, a ripple distribution can still be observed in the sample, even though the center of the membrane still presents lower valleys than
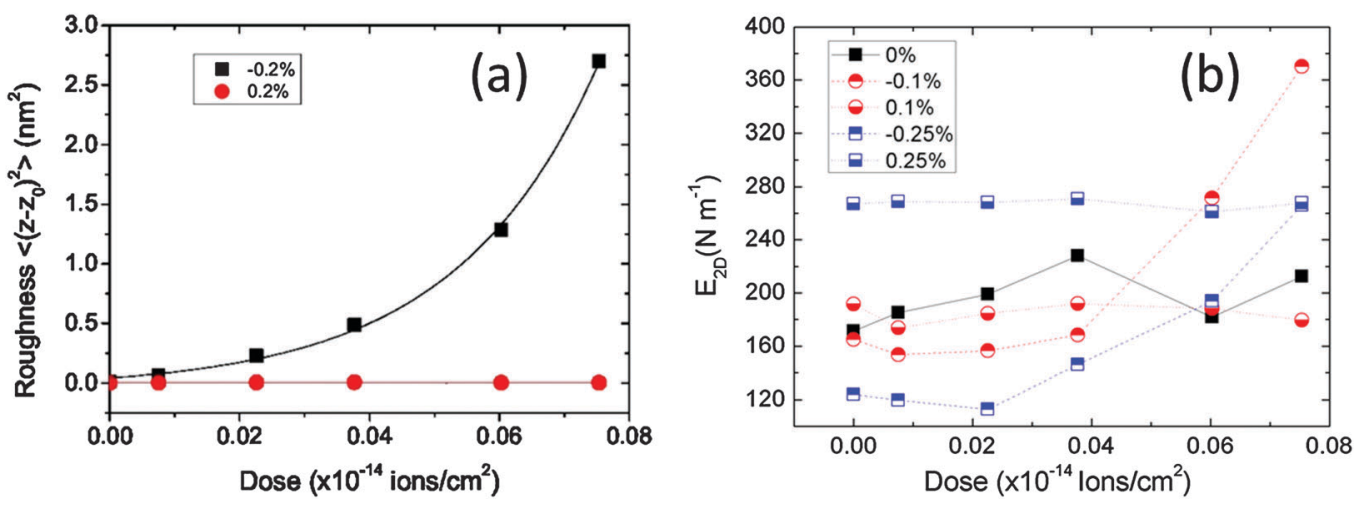

Fig. 3 (a) Roughness as a function of dose for two different strains: $-0.2 \%$ (compressive) and $0.2 \%$ (tensile) and (b) values of $E_{2 D}$ as a function of dose for different applied strains: compressive $-0.25 \%,-0.1 \%$, (squares, half filled) $0 \%$ (squares-filled) and tensile, $+0.1 \%$ and $+0.25 \%$ (circles) . 

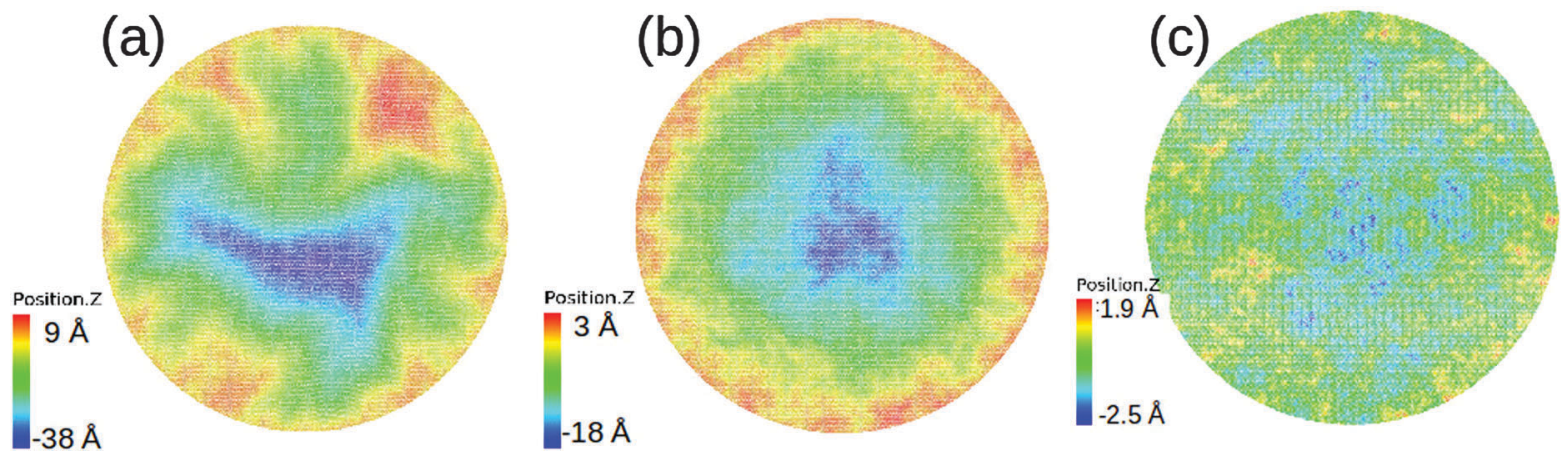

Fig. 4 Values of the $Z$-coordinate (in $\AA$ ) after 1000 ions or a dose of $7.5 \times 10^{12}$ ions per $\mathrm{cm}^{2}$ for a graphene membrane and different applied strains (a) compressive $-0.25 \%$, (b) no strain and (c) tensile $+0.25 \%$.

before irradiation. While the sample is almost unaffected when there is an applied tensile strain of about $0.1 \%$ or higher, the out-of-plane deformations increase for lower strains or compressive strains. This deformation is not symmetrical: the maximum values of $Z$-coordinate follow the same dependence as observed without irradiation, whereas the minimum values of the $Z$-coordinate are highly modified by the irradiation. For example, for a $-0.25 \%$ compression after irradiation, the maximum value of the $Z$-coordinate is $9 \AA$, whereas the minimum value is $-38 \AA$. The initial value of the maximum $Z$-coordinate before irradiation for this same strain is $\sim 3.5 \AA$, as shown in Fig. 2(a). For the case of a tensile strain of $0.25 \%$ the initial maximum value of the $Z$-coordinate before irradiation is $\sim 1.9 \AA$, which after irradiation changes only slightly to a value of $-2.5 \AA$ for the minimum $Z$-coordinate.

These results show that the changes produced by irradiation with respect to the out-of-plane deformation are very different from those induced by strain but they depend significantly on the initial strain state of the membrane. In the same way that a rippled piece of paper can be flattened covering a larger area, the forward momentum of the energetic particles tends to remove the ripple distribution bowing down the sample and producing a deep valley due to the constrained boundaries. Because compressed samples have more ripples available to be flattened by the energetic particles, this effect is particularly efficient in compressed samples. As a result, the topology of the compressed samples after irradiation approaches that of a tensioned sample, provided the curvature of the valley is neglected as a first approximation, and producing a stiffer response of the sample when subjected to nanoindentation.

These results provide an alternative explanation of recent experimental measurements of the $E_{2 \mathrm{D}}$ under irradiation. ${ }^{37}$ These experiments show that at very low doses, such as those studied herein, there is a strong increase in the $E_{2 \mathrm{D}}$, which has been interpreted as the result of defect production. According to López-Polín et al., ${ }^{37}$ vacancies produced by the irradiation quench long range fluctuations resulting in a stiffer membrane. More recent molecular dynamics simulations ${ }^{34}$ have attributed the increase in stiffness to the production of monovacancies. In those simulations, however, vacancies are introduced randomly in the sample instead of being the result of an irradiation and no discussion is included with respect to the temperature of the simulations or out-of-plane displacements. Herein, we give another interpretation of the effect of irradiation on the elastic properties of a membrane; the changes in roughness induced by irradiation (and not just defect production) are responsible for the changes in mechanical properties.

\section{Conclusions}

Molecular dynamics simulations of graphene membranes irradiated with $140 \mathrm{eV}$ Ar ions with different initial strains, from $-0.25 \%$ (compression) to $0.25 \%$ (tension) were performed. The defect production rate under these conditions was approximately $80 \%$ for any of the strains studied, with a slightly higher probability of formation of divacancies when applying a compressive strain than a tensile strain. The concentration of defects increased linearly with dose for all cases studied. Ripples existing in a free standing graphene are strongly modified when the membrane is irradiated, and these changes depend on the initial strain applied to the membrane. While the size of these ripples does not change with dose when a tensile strain is applied, under compression, there is a non-linear dependence with dose. Initially, it only changes slightly and then the roughness increases significantly at higher doses. Moreover, these changes in the ripple distribution induced by the irradiation are different from those induced by strain. Strain obtains, under compression, larger ripples with shorter wavelengths, whereas tension results in opposite behavior, smaller ripples and longer wavelengths. As a result, unirradiated samples under compression are softer than without an applied strain, whereas samples under tension are stiffer. After irradiation, the behavior is quite different: compressed samples are stiffer, whereas the samples under tension do not show changes in their mechanical properties. We attribute this difference to the changes in the out-of-plane displacements induced by the irradiation, which flattens these ripples producing a well that is deeper when the membrane is under compression.

These results provide an alternative way to explain the recent experiments of stiffening of graphene membranes under low dose irradiation. Moreover, these simulations point towards 
paths to tailor the mechanical properties of graphene through a combination of applied strain and irradiation.

\section{Author contributions}

J. M.-A. and C. R. contributed equally to this study. They performed the molecular dynamics simulations and analysis, J. M.-A. focused on the irradiation calculations and C. R. on the nanoindentation. M. J. C. wrote the first draft of the manuscript and all authors contributed to the writing, analysis and discussion.

\section{Acknowledgements}

We acknowledge Professors Cristina Gómez-Navarro, Juan José Palacios and Joaquín Fernandez-Rossier for fruitful discussions. The simulations have been carried out in the supercomputer MareNostrum at Barcelona Supercomputing Center - Centro Nacional de Supercomputación (The Spanish National Supercomputing Center), as well as in the computers of the Dept. of Applied Physics at the UA. This study is supported by the Generalitat Valenciana through grant reference PROMETEO2012/ 011 and the Spanish government through grant FIS2010-21883. CJR and EMB thanks support from SeCTyP-UNCuyo grant M003, and ANPCyT grant PICT-2014-0696. CJR thanks CONICET and the 310 Group at FCEN-UNCuyo.

\section{References}

1 K. Geim and K. S. Novoselov, Nat. Mater., 2007, 6, 183-191.

2 K. S. Novoselov, Z. Jiang, Y. Zhang, S. V. Morozov, H. L. Stormer, U. Zeitler, J. C. Maan, G. S. Boebinger, P. Kim and A. K. Geim, Science, 2007, 315, 1379.

3 S. Ghosh, I. Calizo, D. Teweldebrhan, E. P. Pokatilov, D. L. Nika, A. A. Balandin, W. Bao, F. Miao and C. N. Lau, Appl. Phys. Lett., 2008, 92, 151911.

4 J. Hu, X. Ruan and Y. P. Chen, Nano Lett., 2009, 9, 2730-2735.

5 Y. W. Son, M. L. Cohen and S. G. Louie, Nature, 2006, 444, 347-349.

6 F. Bonaccorso, Z. Sun, T. Hasan and A. C. Ferrari, Nat. Photonics, 2010, 4, 611-622.

7 C. Lee, X. Wei, J. W. Kysar and J. Honer, Science, 2008, 321, 385-388.

8 L. A. Ponomarenko, F. Schedin, M. I. Katsnelson, R. Yang, E. W. Hill, K. S. Novoselov and A. K. Geim, Science, 2008, 320, 356-358.

9 X. Wang, X. Li, L. Zhang, Y. Yoon, P. K. Weber, H. Wang, J. Guo and H. Dai, Science, 2009, 324, 768-771.

10 Y.-M. Lin, C. Dimitrakopoulos, K. A. Jenkins, D. B. Farmer, H.-Y. Chiu and A. Grill, Ph. Avouris, Science, 2010, 327, 662.

11 S. Bae, H. Kim, Y. Lee, X. Xu, J.-S. Park, Y. Zheng, J. Balakrishnan, T. Lei, H. Kim, Y. Song, Y.-J. Kim, K. S. Kim, B. Özyilmaz, J.-H. Ahn, B. Hong and S. Iijima, Nat. Nanotechnol., 2010, 5, 574-578.
12 K. S. Novoselov, A. K. Geim, S. V. Morozov, D. Jiang, Y. Zhang, S. V. Dubonos, I. V. Grigorieva and A. A. Firsov, Science, 2004, 306, 666-669.

13 S. M. Chowdhury, G. Lalwani, K. Zhang, J. Y. Yang, K. Neville and B. Sitharaman, Biomaterials, 2013, 34, 283-293.

14 Q. He, H. G. Sudibya, Z. Yin, S. Wu, H. Li, F. Boey, W. Huang, P. Chen and H. Zhang, ACS Nano, 2010, 4, 3201-3208.

15 F. Torrisi, T. Hasan, W. Wu, Z. Sun, A. Lombardo, T. S. Kulmala, G.-W. Hsieh, S. Jung, F. Bonaccorso, P. J. Paul, D. Chu and A. C. Ferrari, ACS Nano, 2012, 6, 2992-3006.

16 Industrial \& Specialty Printing, http://industrial-printing. net/content/developments-conductive-inks\#.Vp5vNJrhBdg, accessed January 2016.

17 A. Hashimoto, K. Suenaga, A. Gloter, K. Urita and S. Iijima, Nature, 2004, 430, 870-873.

18 M. M. Ugeda, I. Brihuega, F. Guinea and J. M. GómezRodríguez, Phys. Rev. Lett., 2010, 104, 096804.

19 H.-M. Chien, M.-C. Chuang, H.-C. Tsai, H.-W. Shiu, L.-Y. Chang and C.-H. Chen, Carbon, 2014, 80, 318-324.

20 J. Martinez-Asencio and M. J. Caturla, Nucl. Instrum. Methods Phys. Res., Sect. B, 2015, 352, 225-228.

21 W. Li, L. Liang, S. Zhao, S. Zhang and J. Xue, J. Appl. Phys., 2013, 114, 234304.

22 C. Jin, F. Lin, K. Suenaga and S. Iijima, Phys. Rev. Lett., 2009, 102, 195505.

23 S. Zhao, J. Xue, Y. Wang and S. Yan, Effect of $\mathrm{SiO}_{2}$ substrate on the irradiation-assisted manipulation of supported graphene: a molecular dynamics study, Nanotechnology, 2012, 23, 285703.

24 W. Li, X. Wang, X. Zhang, S. Zhao, H. Duan and J. Xue, Mechanism of the defect formation in supported graphene by energetic heavy ion irradiation: the substrate effect, Sci. Rep., 2015, 5, 9935.

25 Q. Wang, Y. Shao, D. Ge, Q. Yang and N. Ren, Surface modification of multilayer graphene using $\mathrm{Ga}$ ion irradiation, J. Appl. Phys., 2015, 117, 165303.

26 K. Liu, C.-L. Hsin, D. Fu, J. Suh, S. Tongay, M. Chen, Y. Sun, A. Yan, J. Park, K. M. Yu, W. Guo, A. Zettl, H. Zheng, D. C. Chrzan and J. Wu, Self-Passivation of Defects: Effects of High-Energy Particle Irradiation on the Elastic Modulus of Multilayer Graphene, Adv. Mater., 2015, 27, 6841-6847.

27 Y. Asayama, M. Yasuda, K. Tada, H. Kawata and Y. Hirai, J. Vac. Sci. Technol., B: Nanotechnol. Microelectron.: Mater., Process., Meas., Phenom., 2012, 30, 06FJ02.

28 P. Bellido and J. M. Seminario, J. Phys. Chem. C, 2012, 116, 4044-4049.

29 X.-M. Qin, T.-H. Gao, W.-J. Yan, X.-T. Guo and Q. Xie, J. Phys. Chem. C, 2012, 1061, 19-25.

30 O. Lehtinen, J. Kotakoski, A. V. Krasheninnikov, A. Tolvanen, K. Nordlund and J. Keinonen, Phys. Rev. B: Condens. Matter Mater. Phys., 2010, 81, 153401.

31 J. Kotakoski, C. H. Jin, O. Lehtinen, K. Suenaga and A. V. Krasheninnikov, Phys. Rev. B: Condens. Matter Mater. Phys., 2010, 82, 113404. 
32 J. Kotakoski, C. Brand, Y. Lilach, O. Cheshnovsky, C. Mangler, M. Arndt and J. C. Meyer, Nano Lett., 2015, 15, 5944-5949.

33 H. Åhlgren, S. K. Hämäläinen, O. Lehtinen, P. Liljeroth and J. Kotakoski, Phys. Rev. B: Condens. Matter Mater. Phys., 2013, 88, 155419.

34 L. Tapasztó, G. Dobrik, P. Nemes-Incze, G. Vertesy, P. Lambin and L. P. Biró, Phys. Rev. Lett., 2008, 78, 233407.

35 C. Gómez-Navarro, M. Burghard and K. Kern, Nano Lett., 2008, 8, 2045-2049.

36 G.-H. Lee, R. C. Cooper, S. J. An, S. Lee, A. van der Zande, N. Petrone, A. G. Hammemberg, C. Lee, B. Crawford, W. Oliver, J. W. Kysar and J. Hone, Science, 2013, 340, 1073-1076.

37 G. López-Polín, C. Gómez-Navarro, V. Parente, F. Guinea, M. I. Katsnelson, F. Pérez-Murano and J. Gómez-Herrero, Nat. Phys., 2014, 11, 26-31.

38 D. G. Kvashnin and P. B. Sorokin, J. Phys. Chem. Lett., 2015, 6, 2384.

39 A. Fasolino, J. H. Los and M. I. Katsnelson, Nat. Mater., 2007, 6, 858.

40 J. C. Meyes, A. K. Geim, M. I. Katsnelson, T. J. Booth and S. Roth, Nature, 2007, 446, 60.

41 S. Lee, Nanoscale Res. Lett., 2015, 10, 422.

42 D. Yoon, Y.-W. Son and H. Cheong, Nano Lett., 2011, 11, 3227.
43 G. Gui, Z. Jianxin and M. Zhenqiang, J. Phys.: Conf. Ser., 2012, 402, 012004.

44 W. Bao, F. Miao, Z. Chen, H. Zhang, W. Jang, C. Dames and C. N. Lau, Nat. Nanotechnol., 2009, 4, 562.

45 S. Plimpton, J. Comp. Physiol., 1995, 117, 1-19.

46 J. Tersoff, Phys. Rev. Lett., 1988, 61, 2879.

47 F. Ziegler, J. P. Biersack and U. Littmark, The Stopping and Range of Ions in Solids, Pergamon Press, New York, 1985.

48 N. Inui, K. Mochiji and K. Moritani, Nanotechnology, 2008, 19, 505501.

49 E. Polak Operationnelle and G. Ribière, Res. Off., 1969, 16, 35-43.

50 A. Stukowski, Modell. Simul. Mater. Sci. Eng., 2010, 18, 015012.

51 L. Kelchner, S. Plimpton and J. C. Hamilton, Phys. Rev. B: Condens. Matter Mater. Phys., 1998, 58, 11085.

52 W. Jiang, J.-S. Wang and B. Li, Phys. Rev. B: Condens. Matter Mater. Phys., 2009, 80, 113405.

53 A. Libai and J. G. Simmonds, The Non-Linear Theory of Elastic Shells, Cambridge University Press, Cambridge, 2nd edn, 1998.

54 U. Komaragiri, M. R. Begley and J. G. Simmonds, J. Appl. Mech., 2005, 72, 203.

55 U. Monteverde, J. Pal, M. A. Migliorato, M. Missous, U. Bangert, R. Zan, R. Kashtiban and D. Powell, Under pressure: control of strain, phonons and bandgap opening in rippled graphene, Carbon, 2015, 91, 266-274. 